\title{
Role of Renin-Angiotensin System, Renal Nerve System, and Oxidative Stress in Chronic Stress-Induced Renal Expression of Aquaporin-1 in Rats
}

\author{
Hu L-X ${ }^{1 \#}$, J iang W-Y" ${ }^{2 \#, ~ W a n g ~ Y-Y 2 \#, ~ C h e n ~ J-W ~}{ }^{3 *}$ \\ and Zhang G-X ${ }^{2 *}$ \\ ${ }^{1}$ Department of Pathophysiology, J ining Medical \\ University, $133 \mathrm{He}$-Hua Road, PR China \\ ${ }^{2}$ Department of Physiology, Medical College of Soochow \\ University, PR China \\ ${ }^{3}$ Department of Internal Medicine, Nanjing University of \\ Chinese Medicine, PR China \\ \#These authors are contributed equally to this work \\ *Corresponding author: Guo-Xing Zhang, \\ Department of Physiology, Medical College of Soochow \\ University, 199 Ren-Ai Road, Dushu Lake Campus, \\ Suzhou Industrial Park, Suzhou 215123, PR China \\ Jing-Wei Chen, Department of Internal Medicine, the \\ Suzhou TCM Hospital Affiliated to Nanjing University \\ of Chinese Medicine, 18 Yang-Su Road, Suzhou 215003, \\ P.R. China
}

Received: June 01, 2021; Accepted: July 02, 2021; Published: July 09, 2021

\begin{abstract}
Aims: To investigate the renal aquaporin-1 (AQP1) expression under chronic stress (induced by foot shock) condition and possible mechanisms involved in rats.

Methods: The chronic stress model was established in male Sprague Dawley (SD) rats by foot shock for two weeks. Rats were randomly divided into control group, chronic stress group, renal denervation group, renal denervation plus chronic stress group, captopril (an angiotensin I converting enzyme inhibitor, ACEI) plus chronic stress group and tempol (a superoxide dismutase mimetic) plus chronic stress group. Body weight, food intake, water intake, blood pressure and heart rate were monitored. Real-time PCR was used to detect the mRNA level of AQP1 in the renal tissue. Immunohistochemistry stain was used to observe the expression and location of AQP1 in rat kidneys.
\end{abstract}

Results: Chronic stress reduced body weight gain and food intake, while it significantly increased systolic blood pressure and renal expressions of mRNA and protein of AQP1 $(\mathrm{P}<0.05)$ as compared with control group. Renal denervation and tempol treatments did not affect stress-induced decreases of body weight gain and food intake. Renal denervation, captopril and tempol treatments decreased systolic blood pressure. Compared with the chronic stress group, mRNA and protein expression of AQP1 was decreased $(P<0.05)$ in renal denervation plus chronic stress group, captopril plus chronic stress group and tempol plus chronic stress group.

Conclusion: Chronic stress induces increase of the AQP1 expression in kidney, which is regulated by renal nerve system, renin-angiotensin system and oxidative stress.

Keywords: Aquaporin-1; Stress-induced hypertension; Oxidative stress; Renal nerve system; Renin-angiotensin system (RAS); Vasopressin

\section{Introduction}

An individual's response to stress should promote the ability of the body to maintain homeostasis. Physiological stress response occurs through several pathways involving the sympathetic nervous system, Hypothalamic-Pituitary-Adrenal (HPA) axis, and behavioral fight-or-flight response. However, chronic stress condition eventually disrupts the homeostasis, resulting in pathological and psychological sequela. The continuous exposure to chronic stress insults can induce maladaptive reactions, including depression, anxiety, cognitive impairment, and cardiovascular diseases [1-3]. Long-term stress affects the functioning of the body in several ways. For instance, overactivation of the autonomic nervous system and abnormal changes in the humoral factors have been reported to be responsible for stress-induced imbalance of the body [4,5], the excessive release of neuronal transmitters and changes in the humoral factors could disturb the homeostasis. Our previous observations have demonstrated that chronic stress (foot shock) activates the neuronal factors through the Renal Sympathetic Nerve System (RSNS), the neuronal and humoral factors through the Renin-Angiotensin System
(RAS), and the humoral factors through oxidative stress. These factors eventually contribute to the development of hypertension $[6,7]$. Therefore, it is imperative to understand how chronic stress disrupts the homeostasis.

Water homeostasis is one of the fundamental regulatory systems in the body. The kidneys are vital for maintaining the water volume of the body. Several Aquaporin (AQP)-type water channels are expressed in this organ to regulate the water volume. AQP1, which is essential for water re-absorption and urinary concentration, has been shown to be abundantly expressed in the proximal tubule, descending thin limb Henle, and vasa recta $[8,9]$. Polyuria has been observed in mice with defective $A Q P 1$, indicating that AQP1 plays an essential role in urine hypertonicity $[10,11]$. AQP1 has also been demonstrated to protect the renal system from endotoxemia-induced acute kidney injury [12], besides retarding the renal cyst development in polycystic kidney disease [13]. Such findings reveal that AQP1 is important in maintaining the renal function and water homeostasis.

Hypertension is a disease involving multiple factors such as genetic, environmental, and lifestyle issues [14-16]. This condition 
increases the cardiovascular and cerebral events [17,18]. The expression of AQP1 in the kidneys varies from one hypertension model to another. For example, high expression of AQP1 has been noted in spontaneous [19] and angiotensin II-induced hypertension models [19], while low expression has been observed in Deoxycorticosterone Acetate (DOCA)-salt [20] and sodium-sensitive hypertension models [21]. The role of AQP1 and its contribution to the development of hypertension in the models remains largely unknown. Our previous observation revealed that RAS, RSNS and oxidative stress reciprocally potentiate to play important roles in the development of foot shockinduced hypertension [6,7]. Until date, there are no data available on the expression of renal AQP1 in chronic stress-induced hypertension model, and the mechanism that regulates its expression is also unclear.

In the present study, we explored the expression of AQP1 in the foot shock-induced hypertension model and investigated the possible mechanisms behind it.

\section{Methods}

\section{Chronic stress animal model}

For this study, 10-week-old male Sprague-Dawley rats were purchased from the Shanghai Laboratory Animal Center (Shanghai, China). The rats were housed under optimal conditions with standard hygiene at the animal center of the Soochow University. The temperature was maintained at $25^{\circ} \mathrm{C}$ with a $12 / 12$ light-dark cycle. Stress was applied by placing the rats in a foot shock box with a metal grid floor for $4 \mathrm{~h}$. The electric current was controlled using an electronic device (0.15 mA, 5-30 s with random current). The rats' renal nerve was surgically denervated under anesthesia with $10 \%$ chloral hydrate according to previous reports [22]. Foot shock stress was commenced 1 week after the surgery. Captopril (an Angiotensin I-Converting Enzyme Inhibitor [ACEI], $100 \mathrm{mg} / \mathrm{kg} /$ day) or Tempol (a superoxide dismutase mimetic, $10 \mathrm{mg} / \mathrm{kg} /$ day) was intraperitoneally injected daily after the stress exposure. Each group consisted of 15 rats. The parameters of body weight, water and food intake, and Systolic Blood Pressure (SBP) of the rats were monitored. SBP was monitored for 30 minutes every day 2 hours after foot shock during the test period for two-week by tail-cuff method as our previous report [7]. The animals were anesthetized and sacrificed on day 14. Their kidneys were harvested for further analysis. The study was reviewed and approved by the Animal Ethics Committee of Soochow University.

\section{Enzyme-Linked Immunosorbent Assay (ELISA) for vasopressin in the serum}

Serum level of vasopressin was measured using commercially available Arg8-vasopressin ELISA kits (Abcam Inc., Shanghai, China, Catalog No: ab205928). All steps were performed according to the manufacturer's instructions.

\section{Real-time PCR for AQP1 mRNA expression in the renal tissues}

Total RNA was isolated from the renal tissues by guanidinium isothiocyanate-acid phenol extraction and quantified by measuring the absorbance at $260 \mathrm{~nm}$. One microgram of the sample was used for reverse transcription, and the rat AQP1 was determined by realtime PCR (Prism 7000; Applied Biosystems, Foster, California). The primer pairs for rat AQP1 cDNA were forward 5'-GAC CTG ATG CTG TGG CTT CT- $3^{\prime}$ and reverse $5^{\prime}$-GAA TGT GGC TCT CGG
TTC AC-3'. The primer pairs for GAPDH were forward 5'-GGA GAT TAC TGC CCT GGC TCC TA-3' and reverse 5'-GAC TCA TCG TAC TCC TGC TTG CTG-3'. The expression of AQP1 mRNAs was normalized using GAPDH mRNA.

\section{Western blot for AQP1 protein expression in the renal tissue}

Renal tissues were homogenized with RIPA buffer $(50 \mathrm{~mm}$ Tris, $\mathrm{pH}$ 7.0, $150 \mathrm{mM} \mathrm{NaCl}, 1 \%$ Triton-X-100) containing phenylmenthanesulfonyl fluoride (R\&D Systems Inc., Minneapolis, US). Homogenates were centrifuged at $12,000 \times \mathrm{g}$ for 10 minutes at $4^{\circ} \mathrm{C}$. Cell protein were separated by SDS-PAGE and transferred to PVDF membranes (Hybond TM-ECL; Amersham Pharmacia Biotech, Inc.). The membranes were blocked in 5\% nonfat milk in PBS and 0.1\% Tween-20 at room temperature. The blots were then incubated with primary antibody: Anti-AQP1 (1:1000, abcam, Inc., Catalog No: ab15080) or anti-GAPDH (Santa Cruz Biotech, Inc., Catalog No: sc-47724). Then the membranes were incubated for 1 hour with a secondary antibody (HRP-conjugated anti-rabbit IgG, 1:2000, abcam, Inc., Catalog No: ab205718). Excess antibody was washed off with TBS-T three times (15 minutes each) before incubation enhanced chemiluminescent reagent (ECL, R\&D Systems Inc, Minneapolis, USA) for $1 \mathrm{~min}$. Subsequently, the membrane was exposed to X-ray film. Immunoreactive bands were detected by the analysis of X-ray films using the software of Image J. The quantity of target proteins is normalized by GAPDH expression.

Immunohistochemical staining for AQP1 protein expression in the renal tissues

The renal tissues were fixed with $4 \%$ formaldehyde for $48 \mathrm{~h}$, dehydrated, and then embedded in paraffin, and cut into $10-\mu \mathrm{m}$ slices. Anti-AQP1 first antibody (1: 200 dilution, Abcam Inc., Shanghai, China, Catalog No: ab15080) was applied, following which the secondary antibody anti-rabbit IgG (1:2000, Abcam Inc., Shanghai, China, Catalog No: ab205718) was added. The protein expression was observed microscopically (Olympus, Japan). Ten areas close to the renal tubules were randomly selected from each section. The proportion of AQP1 positive cells in the renal tubular epithelium were calculated by the following equation:

Positive rate $(\%)=$ number of positive cells/(positive cells number + negative number)X $100 \%$.

The immunohistochemical score of renal AQP1 expression was evaluated as per the criteria listed in Table 1, and the total score was obtained by adding the score of the positive ratio and that of the tinting intensity.

\section{Statistical analysis}

The SPSS 18.0 software (IBM, Inc., Shanghai, China) was used for statistical analysis. The data were presented as mean \pm standard error mean (S.E.M). The grouped data were analyzed using one-way analysis of variance followed by the Student-Newman-Keuls test or one-way analysis $t$ test. $\mathrm{P}<0.05$ was considered statistically significant.

\section{Results}

Increase in rat body weight, amount of food, and water intake

Our data assert that the body weight did not increase in the 

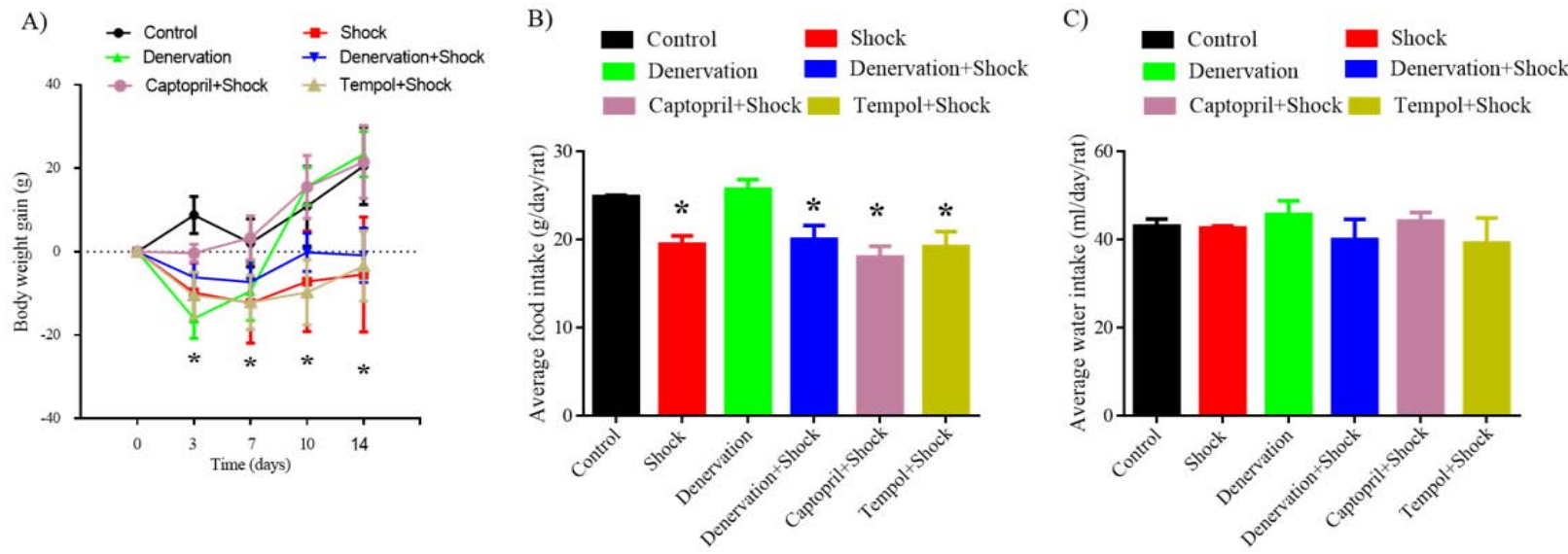

Figure 1: Increase in rat body weight and the amount of food and water intake. (A) Rat body weight gains. (B) Amount of food intake. (C) Amount of water intake. Data are represented as the mean \pm SEM $(n=15)$. ${ }^{*} P<0.05$ compared with the control group.
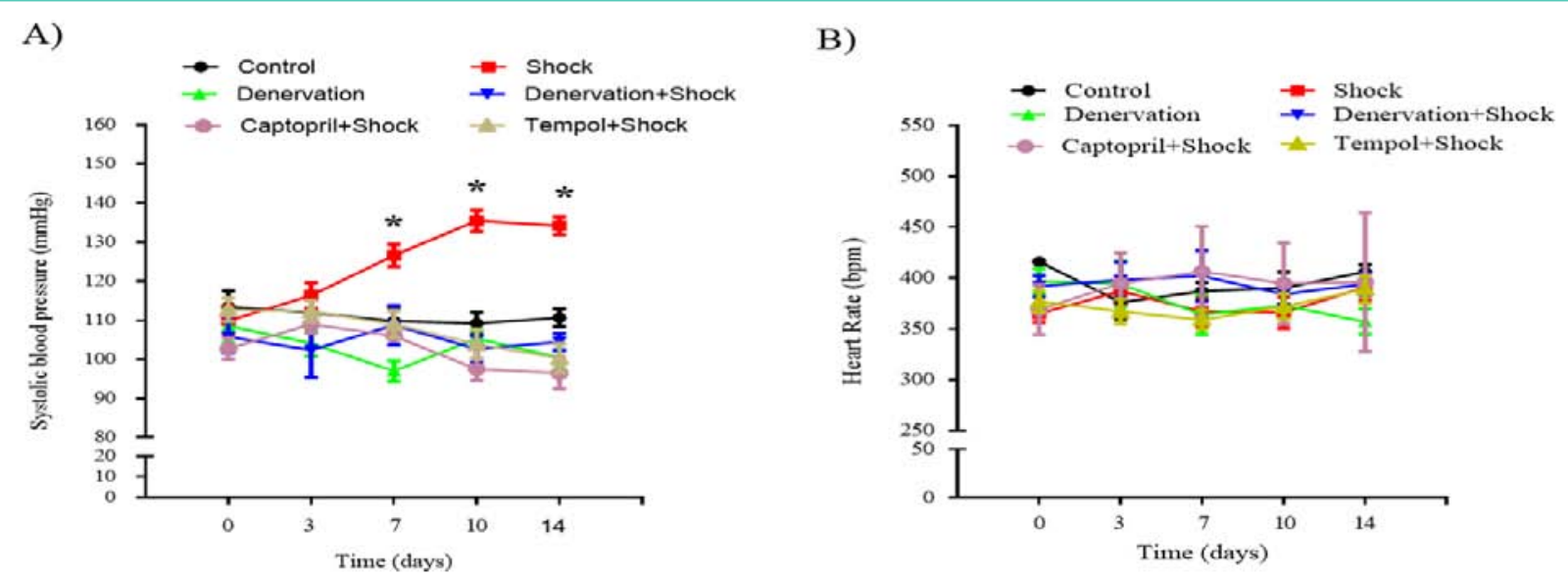

Figure 2: SBP and HR in each group. (A) SBP in each group. (B) HR in each group. Data are represented as the mean \pm SEM ( $n=15)$. ${ }^{*} P<0.05$ vs. SBP on day 0 of the rats subjected to foot shock.

chronic foot shock group $(\mathrm{P}<0.05$ vs. control group, Figure $1 \mathrm{~A})$. Moreover, these findings agree with our previous observations on chronic foot shock stress rat model [7]. The renal denervation group initially exhibited a decrease, followed by recovery to the control level in body-weight gain. Except for the captopril treatment group, other groups subjected to chronic shock showed retarded body-weight gain when compared with the control group $(\mathrm{P}<0.05$, Figure $1 \mathrm{~A})$. The data regarding the amount of food intake implies that the rats subjected to the foot shock consumed less food when compared with those in the control group $(\mathrm{P}<0.05$, Figure $1 \mathrm{~B})$. No difference in water intake was observed among the groups $(\mathrm{P}>0.05$, Figure $1 \mathrm{C})$.

\section{SBP and Heart Rate (HR)}

Chronic foot shock gradually increased the SBP. The SBP was significantly elevated on days 7,10 , and 14 when compared with those taken on day $0(120.1 \pm 4.1,134.8 \pm 3.5,133.5 \pm 7.5$ vs. $109.9 \pm 3.8$ $\mathrm{mmHg}$, respectively, $\mathrm{P}<0.05)$. Renal denervation decreased the SBP on day 7 when compared with the value taken on day $0(97.0 \pm 2.6$ vs. $108.56 \pm 4.3 \mathrm{mmHg}$, respectively, $\mathrm{P}<0.05)$. The rats with renal denervation subjected to foot shock did not exhibit an increase in SBP on days 7,10 , and 14 when compared with that on day 0 (108.5 \pm 5.0 , $102.6 \pm 3.3,104.5 \pm 2.2$ vs. $108.56 \pm 4.3 \mathrm{mmHg}$, respectively, $\mathrm{P}>0.05$ ).
Table 1: Immunohistochemical score criteria.

\begin{tabular}{|c|c|c|c|}
\hline Positive ratio (\%) & Score & Tinting intensity (color) & Score \\
\hline 0 & 0 & Non-staining & 0 \\
\hline$\leq 25$ & 1 & Light color & 1 \\
\hline $26-50$ & 2 & Claybank & 2 \\
\hline $51-75$ & 3 & Sepia & 3 \\
\hline$>75$ & 4 & & \\
\hline
\end{tabular}

The rats treated with captopril did not display an increase in SBP on days 7,10 , and 14 when compared with of the SBP on day 0 in response to chronic foot shock $(106.0 \pm 1.2,97.5 \pm 2.7,96.5 \pm 4.4$ vs. $102.8 \pm 2.6 \mathrm{mmHg}$, respectively, $\mathrm{P}>0.05)$. Those treated with tempol also did not show any elevation in SBP on days 7,10 , and 14 when compared with the value on day $0(109.0 \pm 3.4,103.9 \pm 3.8,100.3 \pm 3.7 v s$. $112.7 \pm 7.5 \mathrm{mmHg}$, respectively, $\mathrm{P}>0.05$ ) (Figure $2 \mathrm{~A}$ ).

Our study did not notice any difference in the HR after the rats were subjected to foot shock in any of the groups (Figure 2B).

\section{AQP1 expression in the renal tissues}

In the renal tissues, the expression of AQP1 mRNA on day 14 was markedly increased in the chronic stress group when compared 


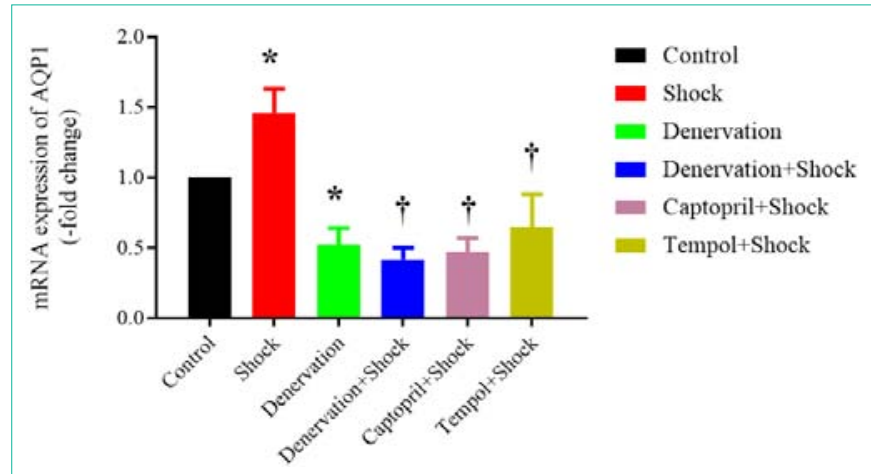

Figure 3: Expression of AQP1 mRNA in the renal tissues $(n=6)$. Data are normalized by control group, which is expressed as 1 . " $P<0.05$ vs. control group. $\uparrow \mathrm{P}<0.05$ vs. shock group.

with that in the control group (1.46 \pm 0.17 -fold, $\mathrm{P}<0.05$ vs. control). Renal denervation lowered the AQP1 mRNA expression in the chronic stress group when compared with that in the control group $(0.52 \pm 0.17$-fold of control, $\mathrm{P}<0.05$ vs. control). In addition, renal denervation abolished the foot shock-induced increase in AQP1 mRNA expression when compared with that in the shock group
(0.42 \pm 0.08 -fold of control vs. $1.46 \pm 0.17$-fold of control, $\mathrm{P}<0.05$ vs. shock). The treatment with captopril also suppressed the chronic shock-induced increase of AQP1 mRNA expression (0.47 \pm 0.10 -fold of control vs. $1.46 \pm 0.17$-fold of control, $\mathrm{P}<0.05 v$ s. shock). In addition, the treatment with tempol inhibited the chronic shock induced increase of AQP1 mRNA expression ( $0.65 \pm 0.23$-fold of control vs. $1.46 \pm 0.17$-fold of control, $\mathrm{P}<0.05$ vs. shock) (Figure 3 ).

The protein expression of AQP1 was observed by western blot and immunohistochemical staining. Data from western blot revealed that chronic shock increased renal AQP1 protein expression compared with that in the control group $(1.96 \pm 0.2$-fold of control, $\mathrm{P}<0.05)$. The denervation itself decreased renal AQP1 protein expression when compared with that in the control group $(0.42 \pm 0.12$-fold of control, $P<0.05)$, renal denervation eliminated foot shock-induced increase of AQP1 protein expression $(0.52 \pm 0.08$ vs. $1.96 \pm 0.2 \%, \mathrm{P}<0.05$ vs. shock). Treatment with captopril or tempol suppressed chronic shock-induced increase of renal AQP1 protein expression (1.07 \pm 0.1 , $0.75 \pm 0.23$ vs. $1.96 \pm 0.23 \%, P<0.05$ vs. shock) (Figure $4 \mathrm{~A}$ ).

Immunohistochemical staining data confirmed that chronic shock enhanced the renal percent of AQP1 positive cells when
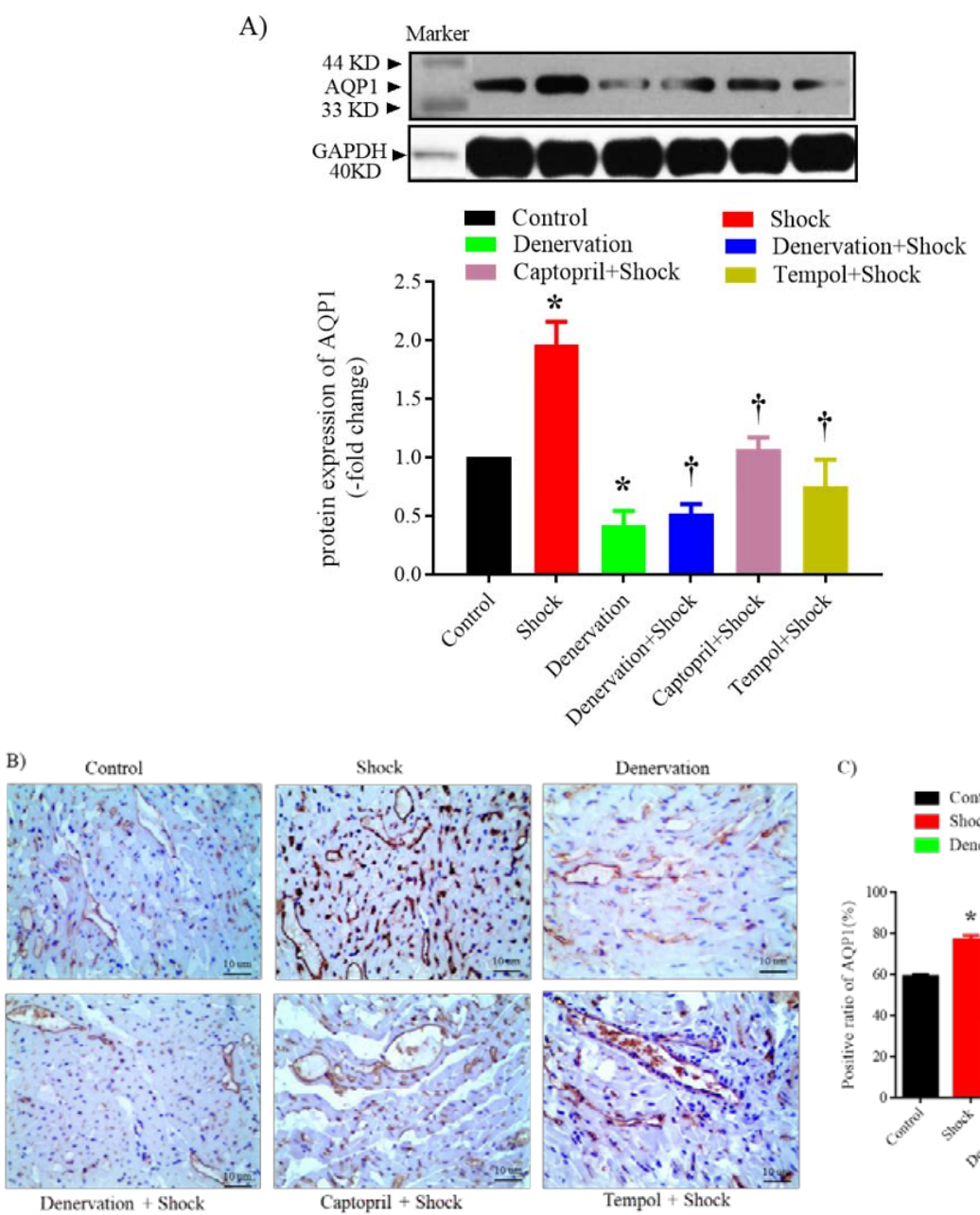

c)
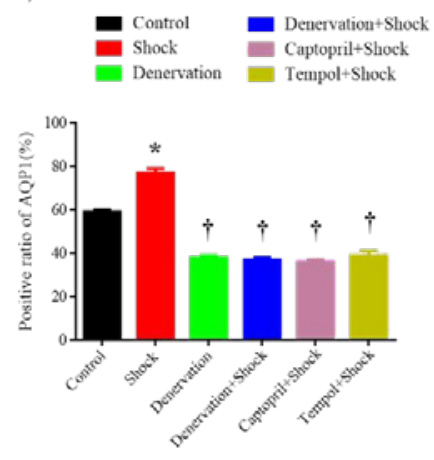

Figure 4: Western blot and Immunohistochemical staining of AQP1 in the renal tissues. A) Protein expression of AQP1 in each group, upper: representative blots of AQP1 and GAPDH, down: densitometry analysis of AQP1 and GAPDH. $n=6$ in each group. B) Representative image of immunohistochemical staining of AQP1 protein in each group. $n=3$ in each group. C) Data analysis of percent of AQP1-positive cells in each group. ${ }^{\star} \mathrm{P}<0.05$ vs. control group. ${ }^{\dagger} \mathrm{P}<0.05$ vs. the shock group. 
Table 2: The renal AQP1 expression level in SD rats.

\begin{tabular}{|c|c|c|c|c|c|}
\hline Groups & Control & Shock & Denervation & Denervation + shock & Captopril + shock \\
\hline Score of positive ratios & 3 & 4 & 2 & 2 & 2 \\
\hline Immuno-reactive score & 2 & 2 & 1 & 1 & 1 \\
\hline Total score & 5 & 6 & 3 & 3 & 3 \\
\hline
\end{tabular}

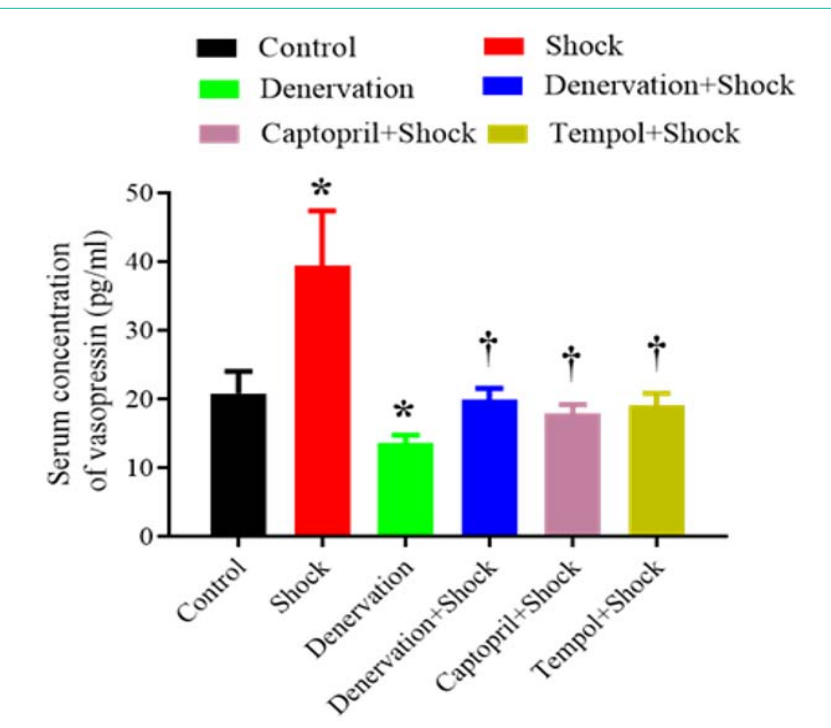

Figure 5: Serum level of vasopressin in each group $(n=6) .{ }^{*} P<0.05$ vs. control group. $† \mathrm{P}<0.05$ vs. the shock.group.

compared with that in the control group $(77 \pm 2 \% v s .59 \pm 1 \%, \mathrm{P}<0.05)$. The denervation decreased the renal percent of AQP1-positive cells when compared with that in the control group $(38 \pm 1 \% v s .59 \pm 1 \%$, $\mathrm{P}<0.05)$. The process abolished foot shock-induced increase in the renal percent of AQP1-positive cells when compared with that in the foot shock group $(37 \pm 1 \% v s .77 \pm 2 \%, \mathrm{P}<0.05 v$ s. shock). The treatment with captopril also suppressed the chronic shock-induced increase in the renal percent of AQP1-positive cells when compared with that in the foot shock group $(36 \pm 1 \%$ vs. $77 \pm 2 \%, \mathrm{P}<0.05$ vs. shock). Moreover, treatment with tempol also inhibited the chronic shock-induced increase in the renal percent of AQP1-positive cells when compared with that in the foot shock group $(39 \pm 2 \%$ vs. $77 \pm 2 \%, \mathrm{P}<0.05$ vs. shock) (Figure 4B, 4C).

The data from immunohistochemical studies revealed that chronic foot shock increased the score of renal AQP1 expression, while renal denervation reduced the score. The animals subjected to denervation, captopril, and tempol treatments did not exhibit any increase in the renal AQP1 expression level (Table 2).

\section{Serum level of vasopressin}

Serum level of vasopressin was increased by chronic foot shock when compared with control group $(20.9 \pm 3.2 \mathrm{pg} / \mathrm{ml} v s .39 .5 \pm 8.0 \mathrm{pg} /$ $\mathrm{ml}, \mathrm{P}<0.05)$. Renal denervation decreased serum vasopressin level compared with control group $(13.7 \pm 1.1 \mathrm{pg} / \mathrm{ml} v s .20 .9 \pm 3.2 \mathrm{pg} / \mathrm{ml}$, $\mathrm{P}<0.05)$, denervation subjected to chronic foot shock group showed lower serum vasopressin level when compared with that of in shock group $(20.0 \pm 1.6 \mathrm{pg} / \mathrm{ml}$ vs. $39.5 \pm 8.0 \mathrm{pg} / \mathrm{ml}, \mathrm{P}<0.05)$. Treatment with captopril or tempol suppressed chronic shock-induced increase of serum level of vasopressin $(18.0 \pm 1.3 \mathrm{pg} / \mathrm{ml}, 19.2 \pm 1.7 \mathrm{pg} / \mathrm{ml} v s .39 .5 \pm$

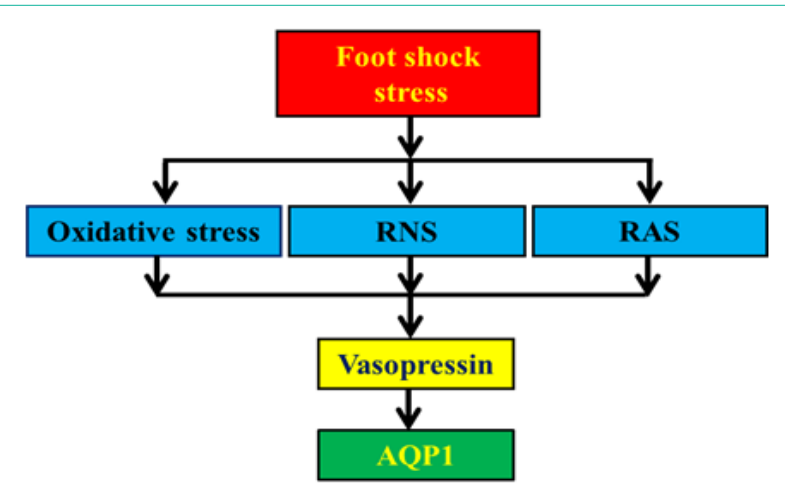

Figure 6: Schematic illustration of the mechanism for foot shock-induced renal expression of AQP1.

$8.0 \mathrm{pg} / \mathrm{ml}, \mathrm{P}<0.05$ vs. shock) (Figure 5).

\section{Discussion}

The present study revealed that chronic stress induces the development of hypertension and is accompanied by an upregulation of renal AQP1 expression, which is mediated by RNS, RAS, and oxidative stress via vasopressin.

Approximately $65-70 \%$ of the water is reabsorbed by AQP1 in the renal proximal tubule, descending thin limb Henle, and vasa recta-the regions in which AQP1 is highly expressed [8]. Based on the primary function of AQP1, a hike in its expression in the renal tissue results in more water being reabsorbed by the kidneys. Therefore, the amount of water released from the body is reduced, which in turn increases the volume of interstitial fluid and finally results in increased blood volume. Earlier clinical studies have reported that sustained hypertension is related to an elevation in the blood volume relative to the capacity of the circulatory system [23]. Enhanced expression of AQP1 was observed in the present study; however, we did not notice any increase in the blood volume of chronically stressed rats based on the reduction in the body weight (Figure 1A). Therefore, we speculate that the increase in water reabsorption caused by elevated AQP1 does not significantly raise the blood volume. Hence, it could not be the cause of increased blood pressure in our model. At present, there is no evidence to demonstrate that a rise in AQP1 contributes to the development of high blood pressure in any hypertensive model.

It is already known that AQP1 is mainly mediated by vasopressin, besides AQP2 [24]. Our previous observations led to an understanding that the mRNA expression of vasopressin in the cerebral hypothalamus is increased by chronic foot shock stimulation [7]. Our present study further demonstrated that chronic foot shock increased serum level of vasopressin (Figure 5). Therefore, our present observation is consistent with a previous report [24]. Furthermore, our earlier findings established that RAS regulates the increase in the mRNA expression of vasopressin [7]. Hence, foot shock induced 
increase in the AQP1 expression should also be regulated by RAS. We had already demonstrated that foot shock stress activates RAS [7], which is potentiated by oxidative stress. Hence, the application of antioxidant alleviates the foot shock induced activation of RAS [6]. Our present data illustrate that antioxidant treatment could inhibit foot shock induced increase of AQP1 expression, thereby suggesting that oxidative stress contributes to the hike in AQP1 expression.

The renal nerve system has been implicated in the regulation of AQP1 in the kidneys. Therefore, the expression of AQP1 proteins was compromised in the denervated kidneys [25]. Our present study has also proved that denervation of the renal nerve lowers the AQP1 expression, which is concordant with a previous observation [25]. Denervated rats did not exhibit an increase in the AQP1 expression in response to chronic foot stress, suggesting that the activation of RNS is another mechanism by which the AQP1 expression was upregulated in the present model.

To confirm by which mechanism RAS, oxidative stress and RNS regulate renal AQP1 expression, we observed serum level of vasopressin since it has been demonstrated to regulate AQP1 expression [24]. All of treatments suppressed serum level of vasopressin in response to chronic foot shock, although our present study did not apply vasopressin antagonist, based on previous literature, it could be speculated that vasopressin mediates RAS, oxidative stress and RNS regulated renal AQP1 expression.

However, it should be noted that our study only focused on the renal AQP1 expression, and other subtypes were not analyzed. Moreover, we did not collect the urine sample, findings from which could have provided more solid data to support the role of AQP1 in the regulation of water homeostasis and its contribution to the development of hypertension. Furthermore, we were unable to explain the possible role of the increased expression of AQP1 based on its function of water reabsorption in the kidneys. The phenomenon might have augmented the water reabsorption and reduced the water secretion, which could have raised the blood pressure synergistically with vasopressin by increasing the circulatory fluid volume. Incorporating the genetic modification of renal AQP1 expression in this model may explain its physiological or pathophysiological function.

Based on previous [26,27] and current observations, we conclude that chronic foot shock stress induces elevated AQP1 expression in the kidneys via the renal sympathetic nerve system, renin-angiotensin system, and oxidative stress by regulation of vasopressin level. These findings have been schematized in Figure 6 .

\section{Declaration}

\section{Ethics approval and consent to participate}

The animal work was taken place at Soochow University and experimental scheme was approved by the Animal Protection and Use Ethics Committee of Soochow University.

\section{Funding}

This work was supported by the Supporting Fund for Teachers' research of Jining Medical University. the National Natural Science Foundation of China (81970422), Suzhou Science and Technology Development Project (SS201745, SS201908), the Gusu Health Human
Resources Training Project (GSWS2019022), and 5th 333 Project of Jiangsu Province (LGY2019012).

\section{Authors' Contributions}

Li-Xun Hu, Wen-Yi Jiang and Yan-Yan Wang performed the experiment.

Jing-Wei Cheng provided part of funding.

Guo-Xing Zhang provided funding and conception.

\section{References}

1. Schneiderman N, Ironson G, Siegel SD. Stress and health: psychological, behavioral, and biological determinants. Annu Rev Clin Psychol. 2005; 1: 607-628.

2. McEwen BS. Protection and damage from acute and chronic stress: allostasis and allostatic overload and relevance to the pathophysiology of psychiatric disorders. Ann N Y Acad Sci. 2004; 1032: 1-7.

3. Chu B, Marwaha K, Ayers D. Physiology, Stress Reaction. StatPearls, Treasure Island, Folorida.

4. Golbidi S, Frisbee JC, Laher I: Chronic stress impacts the cardiovascular system: animal models and clinical outcomes. Am J Physiol Heart Circ Physiol. 2020; 308: H1476-H1498.

5. Wirtz PH, von Kanel R. Psychological Stress, Inflammation, and Coronary Heart Disease. Curr Cardiol Rep. 2017; 19: 111.

6. Dong T, Chen JW, Tian LL, Wang LH, Jiang RD, Zhang Z, et al. Role of the renin-angiotensin system, renal sympathetic nerve system, and oxidative stress in chronic foot shock-induced hypertension in rats. Int J Biol Sci. 2015; 11: 652-63.

7. Wang LH, Dong T, Liu BB, Zhao XD, Chen JW, Murao K, et al. Contribution of the renin-angiotensin system in chronic foot-shock induced hypertension in rats. Life Sci. 2015; 121: 135-144.

8. Nielsen S, Frokiaer J, Marples D, Kwon TH, Agre P, Knepper MA. Aquaporins in the kidney: from molecules to medicine. Physiol Rev. 2002; 82: 205-244.

9. HuaY, Ying X, Qian Y, Liu H, Lan Y, Xie A, et al. Physiological and pathological impact of AQP1 knockout in mice. Biosci Rep. 2019; 39: BSR20182303.

10. Yang B, Ma T, Verkman TS. Erythrocyte water permeability and renal function in double knockout mice lacking aquaporin-1 and aquaporin-3. J Biol Chem. 2001; 276: 624-628.

11. Verkman AS, Yang B. Aquaporin gene delivery to kidney. Kidney Int. 2002; 61: S120-S124.

12. Wang W, Li C, Summer SN, Falk S, Wang W, Ljubanovic D, et al. Role of AQP1 in endotoxemia-induced acute kidney injury. Am J Physiol Renal Physiol. 2008; 294: F1473-F1480.

13. Wang W, Li F, Sun Y, Lei L, Zhou H, Lei T, et al. Aquaporin-1 retards renal cyst development in polycystic kidney disease by inhibition of Wnt signaling. FASEB J. 2015; 29: 1551-1563.

14. Hall JE, do Carmo JM, da Silva AA, Wang Z, Hall ME. Obesity, kidney dysfunction and hypertension: mechanistic links'. Nat Rev Nephrol. 2019; 15: 367-385

15. Zhang JR, Hu WN, Li CY. A Review of the Epidemiological Evidence for Adducin Family Gene Polymorphisms and Hypertension. Cardiol Res Pract. 2019; 2019: 7135604

16. Gupta R, Wood DA. Primary prevention of ischaemic heart disease populations, individuals, and health professionals. Lancet. 2019; 394: 685696.

17. Cohen JB, Lotito MJ, Trivedi UK, Denker MG, Cohen DL, Townsend RR. Cardiovascular Events and Mortality in White Coat Hypertension: A Systematic Review and Meta-analysis. Ann Intern Med. 2019; 170: 853-862.

18. Thakkar HV, Pope A, Anpalahan M. Masked Hypertension: A Systematic Review. Heart Lung Circ. 2020; 29: 102-111. 
19. Lee J, Kim S, Kim J, Jeong MH, Oh Y, Choi KC. Increased expression of renal aquaporin water channels in spontaneously hypertensive rats. Kidney Blood Press Res. 2006; 29: 18-23.

20. Bae EH, Kim IJ, Ma SK, Kim SW. Altered regulation of renal sodium transporters and natriuretic peptide system in DOCA-salt hypertensive rats. Regul Pept. 2009; 157: 76-83.

21. Procino G, Romano F, Torielli L, Ferrari P, Bianchi G, Svelto M, et al Altered expression of renal aquaporins and alpha-adducin polymorphisms may contribute to the establishment of salt-sensitive hypertension. Am J Hypertens. 2011; 24: 822-828.

22. Eriguchi M, Tsuruya K. Renal Sympathetic Denervation in Rats Methods Mo Biol. 2016; 1397: 45-52.

23. Safar ME, London GM, Weiss YA, Milliez PL. Altered blood volume regulation in sustained essential hypertension: a hemodynamic study. Kidney Int. 1975; 8: $42-47$
24. Belkacemi L, Beall MH, Magee TR, Pourtemour M, Ross MG. AQP1 gene expression is upregulated by arginine vasopressin and cyclic AMP agonists in trophoblast cells. Life Sci. 2008; 82: 1272-1280.

25. Lee J, Yoo K, Kim SW, Jung KH, Ma SK, Lee YK, et al. Decreased expression of aquaporin water channels in denervated rat kidney. Nephron Physiol. 2006; 103: 170-178.

26. Antza C, Vazakidis P, Doundoulakis I, Bouras E, Haidich AB, Stabouli S, et al. Masked and white coat hypertension, the double trouble of large arteries: A systematic review and meta-analysis. J Clin Hypertens (Greenwich). 2020; 22: 802-811.

27. Li XC, Shull GE, Miguel-Qin E, Zhuo JL. Role of the Na+/H+ exchanger 3 in angiotensin II-induced hypertension Physiol Genomics. 2002; 47: 479-487. 